\title{
Access to edge scenarios for testing a scraper element in early operation phases of Wendelstein $7-\mathrm{X}$
}

\author{
H. Hölbe ${ }^{a}$, T. Sunn Pedersen ${ }^{a}$, J. Geiger $^{a}$, S. Bozhenkov $^{a}$, R. König ${ }^{a}$, \\ Y. Feng ${ }^{a}$, J. Lore ${ }^{b}$, A. Lumsdaine ${ }^{b}$ and the Wendelstein 7-X Team \\ ${ }^{a}$ Max Planck Institute for Plasma Physics, Wendelsteinstr. 1, 17491 Greifswald, Germany \\ ${ }^{b}$ Oak Ridge National Laboratory, Oak Ridge, 37831-6169, TN, USA
}

\section{Abstract}

The edge topology of magnetic fusion devices is decisive for the control of the plasma exhaust. In Wendelstein $7-X$, the island divertor concept will be used, for which the edge topology can change significantly as the internal currents in a plasma discharge evolve towards steady-state. Consequently, the device has been optimized to minimize such internal currents, in particular the bootstrap current [1]. Nonetheless, there are predicted pulse scenarios where effects of the remaining internal currents could potentially lead to overload of plasma-facing components. These internal currents are predicted to evolve on long time scales (tens of seconds) so their effects on the edge topology and the divertor heat loads may not be experimentally accessible in the first years of $W 7-X$ operation, where only relatively short pulses are possible. However, we show here that for at least one important long-pulse divertor operation issue, relevant physics experiments can be performed already in short-pulse operation, through judicious adjustment of the edge topology by the use of the existing coil sets. The specific issue studied here is a potential overload of the divertor element edges. This overload might be mitigated by the installation of an extra set of plasma-facing components, so-called scraper elements, as suggested in earlier publications.
It is shown here that by a targeted control of edge topology, the effectiveness of such scraper elements can be tested already with uncooled test-scraper elements in short-pulse operation. This will allow an early and well-informed decision on whether long-pulse-capable (actively cooled) scraper elements should be built and installed.

\section{Introduction}

The control of plasma exhaust and its interactions with the material walls are key challenges for magnetic confinement fusion energy systems, see e.g. [2], [3]. The use of the specially designed magnetic topologies that are collectively referred to as diverted configurations, has proven quite effective in meeting this challenge in both tokamaks [4] and stellarators [6], [5]. The challenge is multifaceted and complex; the parts of particular relevance to this work can be summed up as follows: By use of a divertor magnetic topology, can one control the outflow of plasma heat and particles in such a way that the generated neutral particles can be pumped away, the plasma facing components survive the interactions with the outflowing plasma, and the core plasma stays hot, with low impurity content and a controlled density?

Although the stellarator island divertor 
concept [7] chronologically precedes the tokamak divertor by more than a decade, it is much less experimentally explored. It has been tested successfully at modest scales in a few experiments, e.g. in the W7-AS stellarator [5], [8], but its operation at fusion-relevant heat fluxes and pulse lengths still needs to be demonstrated. These tests are planned for the near future in the Wendelstein 7-X (W7-X) stellarator experiment, described in Section 2. The magnetic island topology of W7-X - and any other low-shear stellarator using an island divertor concept - is sensitive to even relatively modest currents, such as those self-generated by the plasma, and the proper and safe operation of the divertor could therefore be compromised. Since W7-X is not equipped with an ohmic transformer, the time evolution of net-current densities in the plasma has to take into account the self-inductance of the plasma which shields plasma current changes initially. Changes in the total plasma current, which are important for the edge topology, thus appear on the time scale of the L/R-time which is in highperformance plasmas (high $n T \tau$ ) in the order of some tens of seconds although the bootstrap current (BSC), the only source in case of no current drive, evolves together with the profiles of temperature and density on the energy confinement time scale. Experimentally this kind of slow time evolution of the total current has been observed in HSX [9] which has also no ohmic transformer.

Therefore BSC effects on the edge topology can only be fully explored in long-pulse operation. However, we will show in this paper, that the relevant long-pulse edge topologies and heat load patterns can be created and studied in short-pulse operation, using the existing, external electromagnets of W7$\mathrm{X}$. Consequently, important physics questions pertaining to long-pulse operation can be addressed in short-pulse operation, i.e. several years before long-pulse operation is possible in W7-X. This ability may be of significant importance for the W7-X program.

We prove this ability by example in this paper: we show that one specific (previously identified) W7-X long-pulse divertor operation issue can be addressed already in shortpulse operation. Our paper is organized as follows:

In Section 2 the presently planned operation phases of Wendelstein 7-X and the steps toward the island divertor are described. The "over-load" problem and the proposed scraper-element (SE) mitigation strategy are reviewed in Section 3. In Section 4, the general strategy is presented; i.e. how to mimic important aspects of long-pulse, high-performance plasma experiments during shorter-pulse, lower-power operation, specifically aspects related to the edge topology and island divertor operation with the help of properly tuned vacuum magnetic configurations. The specific results relative to the scraper-element issue are presented in Section 5 , showing that the functionality of scraper elements can be tested in short-pulse operation. In Section 6 the number of test scraper elements to be installed is analyzed and discussed, and the conclusions are given in Section 7.

\section{Background: W7-X, its operation phases, and its divertor}

W7-X is a modular, optimized stellarator that is about to go into operation in Greifswald, Germany. It aims to demonstrate the fusion reactor relevance of the optimized stellarator concept [10]. High-performance plasma discharges, i.e. high $n T \tau$, are planned with a heating power of currently up to $10 \mathrm{MW}$ ECRH $(140 \mathrm{GHz})$ at a B-field of $2.5 \mathrm{~T}$ for up to $30 \mathrm{~min}$ [11]. One major issue to be stud- 
ied is the efficiency and adequacy of the island divertor concept [12]. This goal will be approached gradually. After an integral commissioning starting in 2015 [13] which only uses a limiter [14], an uncooled divertor will be used in a campaign starting in 2017 for experiments with limited pulse length before the exploration of long-pulse high-power operation will follow from 2020 on using a fully cooled high-heat-flux (HHF) divertor [15]. The TDU and the HHF divertor have the same shape.

The planned operation of W7-X can be divided into separate phases of upgrades of the plasma-facing components to enable quasisteady-state operation. The first plasma phase, the so-called operational phase 1.1 (OP1.1), starts in 2015 with five carbon limiter stripes, and no divertor. In this phase, the heating power and discharge length will be limited so as not to exceed approximately $2 \mathrm{MJ}$ of total injected energy. Afterwards, the limiter is removed and with the completion of the in-vessel installations an uncooled island divertor (Test Divertor Unit or TDU) will be installed [17]. This will be exploited in a oneyear campaign beginning in 2017 in the operational phase 1.2 (OP1.2), the so-called TDU phase. During this phase the heating power is limited to $8 \mathrm{MW}$, and the maximum discharge time depends on the integrated heating power input because of the lack of cooling capability. The discharge time is expected to be on the order of 10 seconds for discharges heated with $8 \mathrm{MW}$. Midway through OP1.2, it is planned to install one or two un-cooled testSEs, to verify their function, and investigate any other impact on discharge performance they may have. The parts of OP1.2 before and after the test-SE installation are referred to as OP1.2a and OP1.2b, respectively.

After OP1.2b (beginning of 2018), the uncooled divertor and the test-SE will be removed and the actively cooled HHF-divertor will be installed, capable of withstanding a steady-state heat-load of $10 \mathrm{MW} / \mathrm{m}^{2}$. Following the installation, operation phase 2 (OP2) is planned to start in 2020 to explore high-performance, quasi-steady-state operation (pulse length up to 30 minutes) with a heating power of up to $10 \mathrm{MW}$, and higher heating power for short pulses.

If the test-SE results are encouraging, i.e. show the necessity and compatibility of such protection elements with good divertor operation, it may be decided to build and install the 10 fully water-cooled steady-state capable SE's. The development of other strategies to deal with this problem will also be pursued. We name here only the development of experimental scenarios by investigating heating scenarios (on- vs off-axis ECRH including ECCD) and/or adjusting the magnetic configuration by further exploiting the flexibility provided by the coil system. However, it falls outside the scope of this article to analyze these here too.

\section{Protection of the diver- tor edge}

In OP1.2 and later, the plasma edge in W7-X is defined by a magnetic island chain that is intersected by divertor plates. The details of the divertor plate, the pumping gap geometry, and the location and size of this edge island chain together determine how and where the convective plasma heat loads are deposited onto the divertor plates, as well as how effectively the resulting neutrals are exhausted. The position of the edge island chain can change due to currents in the plasma, most importantly the MHD equilibrium currents that are directly or indirectly related to the normalized plasma pressure $\beta=2 \mu_{0} p / B^{2}$, as discussed in Section 4. The plasma will therefore affect the edge topology and through that, the divertor operation, in particular in high-performance discharges. 

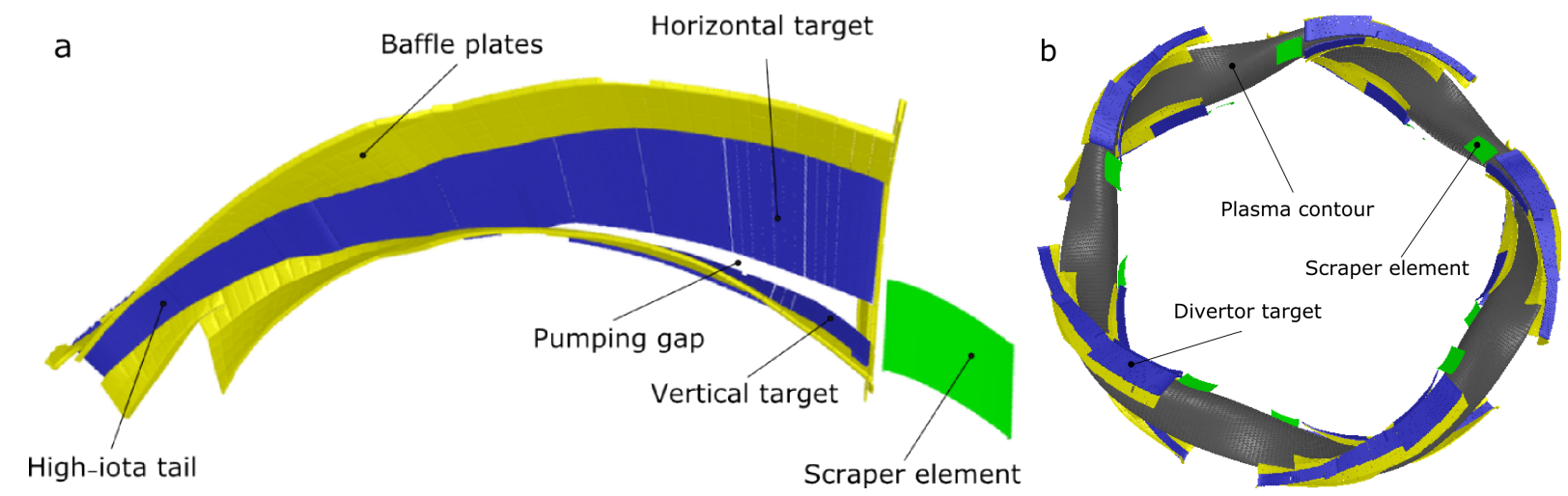

Figure 1: (a) CAD model of one W7-X divertor module as foreseen in OP1.2b with installed scraper element. Target elements are dark (online: blue), baffle plates are light grey (online: yellow) and the scraper element grey (online: green). Around the pumping gap there are the horizontal (upper part) and the vertical (below the pumping gap in the picture) targets. (b) Topview of W7-X Divertor geometry with ten divertor moduls and ten SEs (cf. (a)).
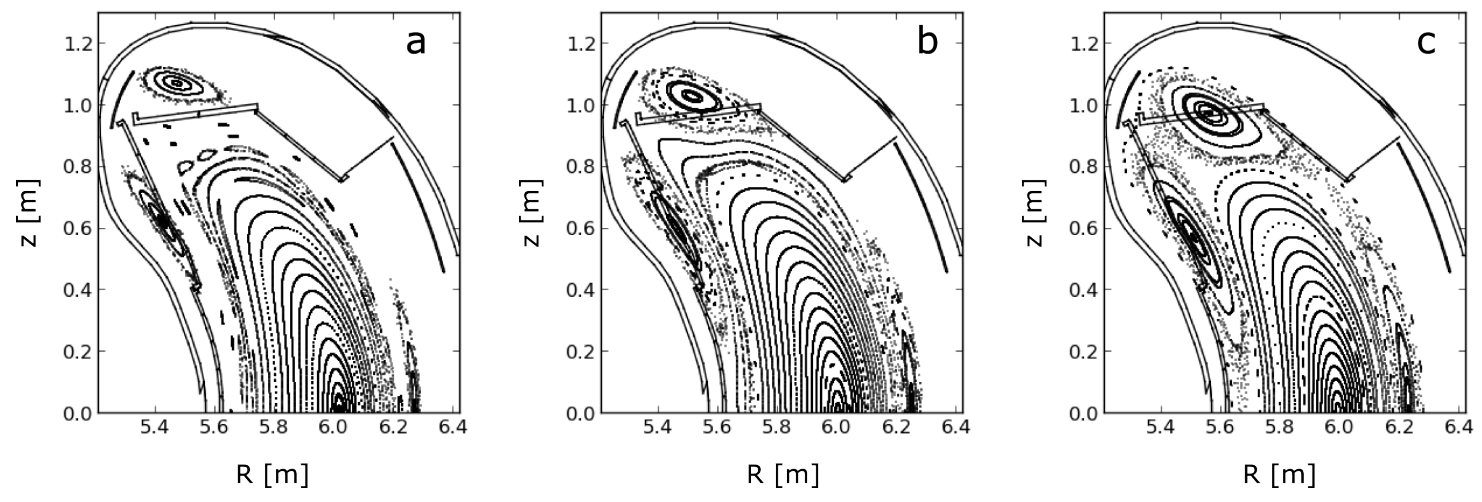

Figure 2: Poincaré plot "SE reference scenario" [18] at $\phi=0^{\circ}$. BCS: (a) $0 \mathrm{kA}$, (b) $22 \mathrm{kA}$ and (c) $43 \mathrm{kA}$. 
Numerical simulations of one of the potentially attractive long-pulse discharge scenarios predict unacceptably high heat loads at the ends of the divertor tiles, near the divertor pumping gap. This high-performance, longpulse scenario is described in detail in [18]. A final bootstrap current of $43 \mathrm{kA}$ is predicted in the simulation and the magnetic configuration is chosen such that the vacuum rotational transform together with the net-current contribution results in the proper island divertor boundary configuration. However, the total plasma current and with it the magnetic edge topology evolves due to the high temperatures on a time scale of several tens of seconds, the $\mathrm{L} / \mathrm{R}$-time. Thus, this time evolution is only accessible in long pulses. The edge topologies for different stages of the evolution of this BC-scenario (BCS) are shown in Figure 2.

In order to avoid this overload the installation of SEs - passive protection elements is under consideration. A SE is an additional target plate placed in front of each divertor module (see Figure 1). It acts to intersect and eliminate (or "scrape off") the plasma parallel heat flux flowing along the magnetic field lines that connect to the critical locations of the HHF divertor at the pumping gap.

This SE concept was described previously in the peer-reviewed literature $[19,20]$. Designed to allow for safe operation of the divertor, it could influence plasma performance negatively - for example, first investigations with EMC3/Eirene shows a significantly reduced pumping efficiency .

In addition, manufacturing, assembly and installation of the needed 10 SEs would require significant resources and time since they would need to be fully water-cooled.

In view of the necessary effort it would be advantageous to test the impact of the SEs on the plasma performance well in advance to allow a timely and well-informed decision on whether to have them manufactured and installed. This, however, requires experimental access to an edge topology similar to that of the scenario that has the heat load problem, the so-called "SE reference scenario".

A test in OP1.2 would also have the benefit that the tests can be performed without the danger of damage to the divertor. This is because the uncooled TDU is installed in this phase which is robust against overload scenarios and free from the danger of water leaks. However, the "SE reference scenario" with its slow time evolution is not accessible during OP1.2: As mentioned, the time limits for full power pulses are on the order of 10 seconds. Numerical calculations indicate that it would take about 40 seconds for the net toroidal current to reach the $22 \mathrm{kA}$ where the problem is most prominent. It is also not guaranteed that the plasma density and heating power will be sufficient in OP1.2 to reach the parameters needed for the "SE reference scenario". In the following we describe how an "SE reference scenario" relevant edge topology can already be generated in OP1.2, allowing the aforementioned experimental test of the potential advantages and disadvantages of an SE installation on the plasma performance.

Before proceeding, a peculiarity of the boundary topology of W7-X should be pointed out which arises in stellarators generally because of their periodicity. Structures (e.g. islands) which are resonant to a rational value of the rotational transform, i.e. comply to $t=n / m$, will close onto themselves after running around $\mathrm{n}$ times poloidally and $\mathrm{m}$ times toroidally, where $\mathrm{m}$ and $\mathrm{n}$ are the smallest numbers forming the rational value. In W7-X, where the so-called standard configuration has a boundary- $t=1$, this means that boundary islands close onto themselves after one toroidal and one poloidal turn. However, assuming strict 5-fold periodicity implies that a structure closing onto itself after one toroidal and one poloidal turn must be present five times. Because of this, five 
independent islands are forming the separatrix at the boundary rotational transform of $t=1$ which is sometimes emphasized by writing $t=5 / 5$.

\section{Development of test scenarios}

The approach taken here is to match the edge topology of the different stages of the evolution of the net plasma current by adjusting the coil currents. That is, the same edge magnetic topology is mimicked as closely as reasonably possible in a zero- $\beta$, zero-toroidalcurrent (i.e., vacuum) configuration as would exist in a high- $\beta$, finite-toroidal-current equilibrium using the flexibility provided by the main and auxiliary coil systems of W7-X [11]. Because the heat load distribution on the different divertor parts is the property of interest, the "SE reference scenario" will be assessed by a sequence of specially designed vacuum configurations which reproduce the important properties of the heat load distributions of the different stages of the "SEreference scenario". The calculations shown in this paper are assuming attached plasmas. In detached plasmas the parallel heat flux could be decreased considerably. Hence, a perfect match of the magnetic configuration in total is not the goal but to reproduce the effects of the boundary topology changes on the load distribution to the different divertor parts. Thus, a configuration with finite $\beta$ and/or a net-toroidal current is seen as equivalent to a vacuum configuration in this context if their relative heat load distributions on the different divertor parts are the same. A comparison of these heat load distributions will be used to calibrate the coil current changes to mimic the different plasma current effects.

There are two distinct plasma current effects on the configuration in W7-X, and they evolve on different time scales. One is due to the perpendicular diamagnetic current density $\vec{j}_{\perp}$ needed for the basic MHD force balance:

$$
\nabla p=\vec{j}_{\perp} \times \vec{B}
$$

Because in toroidal magnetic configurations this current is not divergence free, there is a parallel current density part connected with $\vec{j}_{\perp}$, the so-called Pfirsch-Schlüter (PS) current, which affects the equilibrium but does not contribute to the net toroidal current. In a tokamak, the sum of the diamagnetic and PS currents does produce a net toroidal current, but in a current-free stellarator it does not [21]. The other one, the net toroidal current, is due to the bootstrap current as well as the induced shielding currents which appear in response to the bootstrap current evolution. The diamagnetic current and the PS current together evolve on the time scale of the changes in $\nabla p$ i.e., on the time scale of the energy confinement time (of order $200 \mathrm{~ms}$ in W7-X) whereas the net toroidal current evolves on the much longer plasma selfscreening time, also known as the $\mathrm{L} / \mathrm{R}$ time, which will be on the order of 20-40 seconds for high performance scenarios. Currents may also be induced in conducting structures surrounding the plasma, e.g. the plasma vessel itself. These decay, however, on time scales of less than $50 \mathrm{~ms}$ in W7-X. Thus, they will not play an important role for the slowly evolving equilibrium effects that are important for the divertor operation, and they will consequently be ignored in the following. We will refer to the effects due to the diamagnetic current and the PS current collectively as $\beta$ effects. The $\mathrm{BC}$ and the self-shielding plasma response to it together entail the net toroidal current effects. Because of the very different time scales on which the $\beta$-effect currents and the net toroidal currents evolve, they will be assessed independently. 

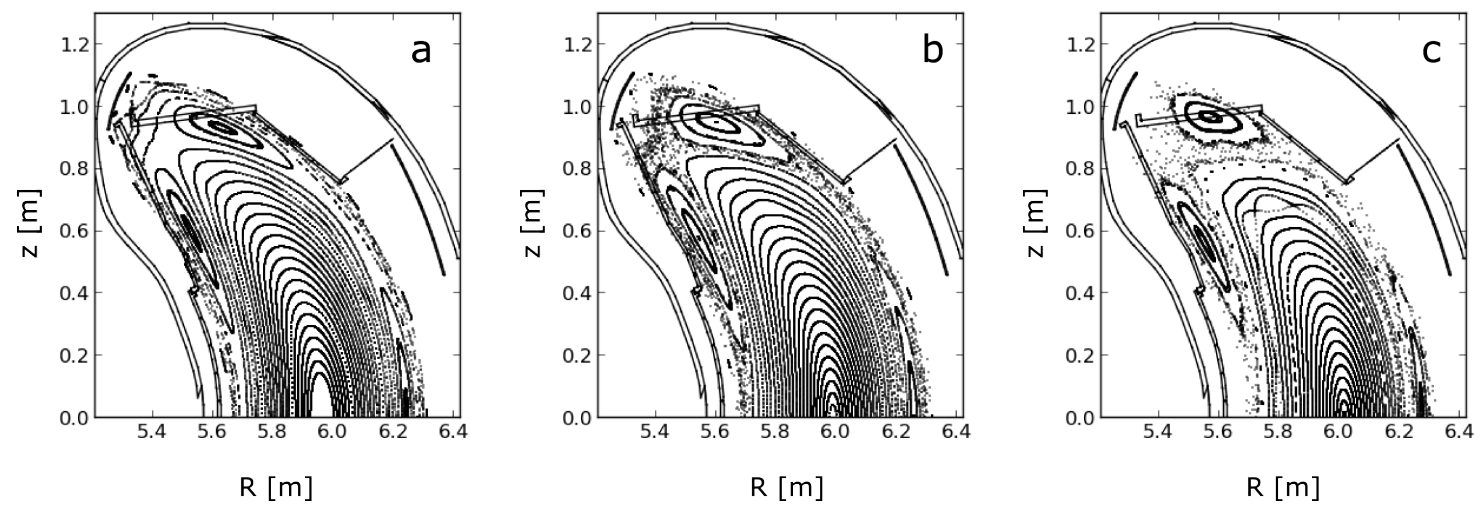

Figure 3: $\beta$ scan of standard configuration (no bootstrap current included). Poincaré plots at $\phi=0^{\circ}$. Average $\beta$ : a: $0.65 \%$, b: $2 \%$ and c: $3.4 \%$. Magnetic fields calculated with VMEC and Extender [22].

\subsection{Effect of plasma $\beta$}

The diamagnetic part of the equilibrium currents is mostly poloidal and its effect on the equilibrium is therefore small since it "competes" with the mostly poloidal current of the main superconducting coils of W7-X. The PS currents generate mainly poloidal field components of the same magnitude as the vacuum field, hence with a stronger effect on the internal flux surfaces (axis shift and change in rotational transform) as well as on the structure of the magnetic field outside the plasma - i.e. the boundary islands. Figure 3 shows the latter effect in a sequence of Poincaré plots of magnetic fields derived from VMEC/Extender [23], [24], [25] calculations [22] with increasing $\beta$ values. Generally, the island width (radial width, not the poloidal extent given by the spacing of the x-points) increases with $\beta$; this can be best observed in the change in the upper island of the bean-shaped cross section. Furthermore, with increasing $\beta$ the field around the $\mathrm{x}$-points/separatrix tends to become stochastic $[25],[26],[27]$.

In order to mimic these $\beta$-effects, neither the 50 non-planar coils nor the 20 planar coils of the main coil system are suited, since changing the currents in these coils tends to change many other important parameters si- multaneously (rotational transform, toroidal mirror field component and/or horizontal plasma position) in addition to the island width.

However, the so-called sweep coils, a set of $5 \times 2$ stellarator-symmetric coils inside the vacuum vessel which all have independent power supplies, can be used in a stellarator symmetric operation mode to control the island size without substantially changing the rest of the topology [28], [29]. This method does not allow a perfect match to the island size at finite $\beta$, but it is sufficiently close enough to generate an interaction between the island chain and the plasma-facing components which is very similar to that at finite $\beta$. This suffices for many purposes, including the ability to mimic the "SE reference scenario". Fig. 4 shows the effect of the sweep coils on the island width for the standard configuration which in this case is mainly reflected by the increase in stochasticity around the boundary islands.

\subsection{Net toroidal current}

The optimization to a near-zero $\mathrm{BC}$ was done for one specific configuration in W7-X. Non-negligible BC exists for other interest- 

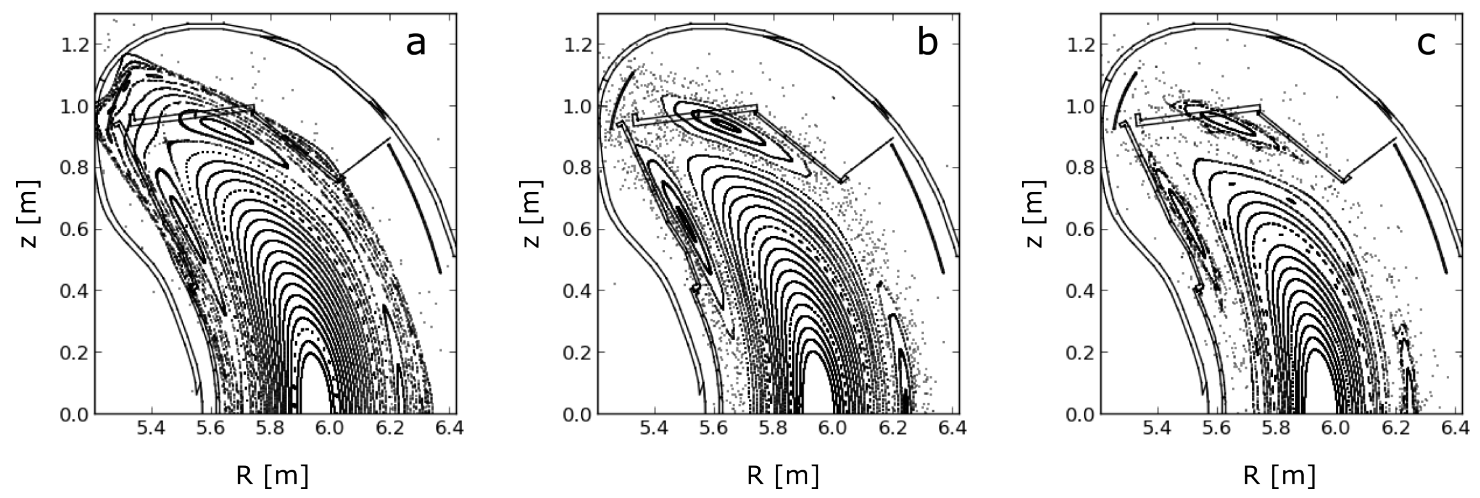

Figure 4: Sweep coil current scan (vacuum configuration). Poincaré plots at $\phi=0^{\circ}$. Vacuum magnetic fields: Modular coil currents $\left(I_{1}=\ldots=I_{5}=1.0\right)$, planar coil currents $\left(I_{A}=I_{B}=0\right)$ and sweep coil currents for island size variation: (a) $I_{s 1}=0, I_{s 1}=0$. (b) $I_{s 1}=-0.02, I_{s 1}=0.02$. (c) $I_{s 1}=-0.04, I_{s 1}=0.04$.

ing configurations and discharge scenarios. Nevertheless, the BC in W7-X is generally an order of magnitude smaller than in a tokamak or classical stellarator of similar size and rotational transform. Due to the low magnetic shear, even this rather small $\mathrm{BC}$ can have a significant impact on the edge islands and the island divertor operation. According to transport simulations for different magnetic configurations achievable in W7-X, configurations with good confinement have usually an increasing bootstrap current [18]. Thus, in order to take advantage of best-confinement configurations, a modest BC might have to be accepted with the result of a changed edge topology and in turn changed heat load patterns on the divertor (see Figure 2 and as discussed in Section 3 ), even if this requires the development of mitigation strategies to allow for safe divertor operation. The net toroidal current first and foremost affects the rotational transform. One of the main purposes of the 20 planar coils is to change the rotational transform $\iota$, without strongly affecting the other properties of the equilibrium. These coils can therefore be used to mimic the effect of a net-toroidal current when none is present. The achievable match is not perfect; the changes in magnetic shear, $d \iota / d \psi$, cannot be matched while simultaneously matching the changes in $\iota$ at the plasma edge $(\psi$ is the toroidal magnetic flux). Nevertheless, for the configurations studied here the effect due to the mismatch in shear is negligible.

Figure 5 shows the movement of the boundary islands with respect to the divertor when tuning the rotational transform up and down from its boundary value of 1.0 in the standard configuration (same currents in the modular coils, no other coils used), using the planar coils. We compare this sequence with VMEC/EXTENDER calculations accounting only for the net-toroidal current to change the boundary island location, i.e. $\beta \equiv 0$. As seen in Fig. 5 the island location changes are reproduced quite well.

Thus, broadly speaking, vacuum field configurations are expected to be able to mimic configurations with finite $\beta$ and finite net toroidal current by jointly adjusting the currents in the sweep coils and the planar coils.

\subsection{Calibration}

In order to be able to determine the coil currents properly so that the vacuum or low- $\beta$ configurations are equivalent in the previously mentioned definition, i.e. equivalence of heat 

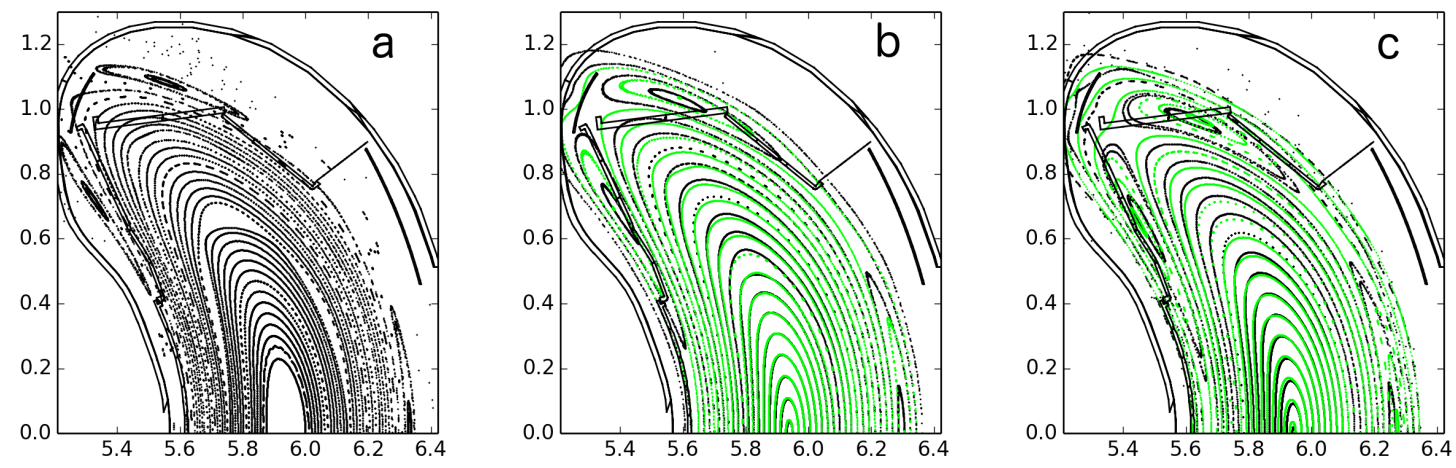

Figure 5: Black: Poincaré plot of iota scan (vacuum configurations) at $\phi=0^{\circ}$. Modular coil currents $\left(I_{1}=\ldots=I_{5}=1.0\right)$ and planar coil currents $\left(I_{A}, I_{B}\right)$ for controlling the rotational transform: (a) $I_{A, B}=$ 0.12. (b) $I_{A, B}=0.07$. (c) $I_{A, B}=0.032$. Green: VMEC fields without beta effect but with net toroidal current: (b) $I_{\text {tor }}=22 \mathrm{kA}$. (c) $I_{\text {tor }}=43 \mathrm{kA}$

load pattern distribution on the different divertor parts, we need to provide a mapping. Thus we assess the relative heat loads on the different divertor parts (targets, baffles, SE) in a global way, i.e. not the local distribution on these parts. To model this, we use a field line diffusion approach that simulates the transport perpendicular to the magnetic field [30]. For this, field lines, starting at the separatrix, are traced. After a random distance a random step perpendicular to the field line is performed and the tracing is continued starting from the new point. If a component (for example the divertor target plate) is hit the point of impact is recorded and the field line tracing procedure is started again with a new point at the separatrix. The number of impact points per area divided by the total number of field lines traced is used as a figure of merit for the fraction of the heat load hitting this area. Later, in Section 5, a cross-check will be done by inspecting the local strike line patterns.

The coil current values given in the following are given as fractions of the maximum current of the respective coil type. Engineering details of the different coil types are not directly relevant to the work presented here, but can be found in [32]. Thus, the so-called standard configuration has the same relative currents for the five modular coils and zero for all others. A configuration is described by the full set of relative coil current values, i.e. for all modular coils, planar coils and control coils.

\subsection{1 $\beta$ calibration}

Figure 6 compares the distribution of the heat loads on the different divertor components (including the SE) resulting from a $\beta$ sequence of MHD-equilibria with the ones resulting from vacuum fields obtained by properly adjusting the currents in the sweep coils. The figure comprises the results of the field line diffusion approach to estimate the heat load distribution for different magnetic fields : several MHD-equilibrium fields (shown as crosses, triangles and circles) and a larger number of vacuum configurations (results shown as lines). The "component load factor" shows the distribution of the heat load, e.g. divertor: 0.8 , SE: 0.2 means $80 \%$ of the total heat load is distributed to the divertor units and $20 \%$ to the SE. The sum of the "component load factor" for the baffle, divertor and SE is 1.0 for all shown cases, which means no other in-vessel components 


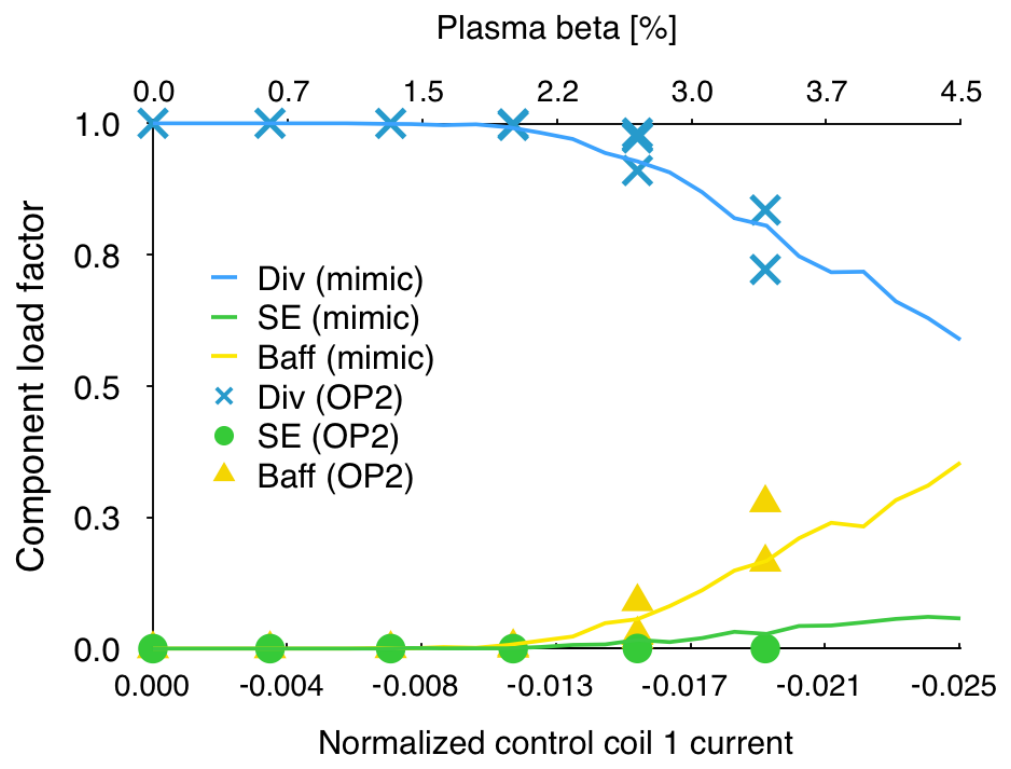

Figure 6: Mimicking plasma beta effect with magnetic coils. Lines: Control coil current scan (vacuum configurations). Crosses/Circles/Triangles: MHD-equilibrium calculation for different beta values. Each equilibrium calculation was done for different values of plasma radius. The y-axis shows the fraction of the heat load going to the various components for the different configurations. The current of control coil 2 is minus the current of control coil 1 . Currents modular coils $=1$, planar coils current $=0$. To achieve a main field of $2.5 \mathrm{~T}$ the normalized currents have to been multiplied by $1.47 \mathrm{MA}$.

are loaded in the shown configurations. As seen, the redistribution of the heat loads with $\beta$ can be well reproduced by an appropriate adjustment of the sweep coil currents.

Note, the "SE reference scenario" has a $\beta$ value of $2.7 \%$. The sweep coil current to imitate this $\beta$-value is used later for the OP1 mimic configurations .

\subsubsection{Iota calibration}

Finite current in the planar coils is used in vacuum configurations to imitate the effect of the net-toroidal current. Figure 7, shows the change of the heat loads on the different components for the net-toroidal current sequence in the "SE reference scenario" as well as for the vacuum configurations obtained by adjusting the currents of the planar coils. With an appropriate mapping of the values of the coil current to the ones of the net-toroidal current a remarkably good match of the heat loads can be achieved.
Fig. 8 compares with the help of Poincaré plots the joint effect of mimicking $\beta$ - and net-toroidal current in the vacuum configurations with the MHD-equilibrium fields derived from VMEC/EXTENDER for the 2 cases with $22 \mathrm{kA}$ and $43 \mathrm{kA}$. Note that there is no perfect agreement in the flux surface geometry (especially in the core the Shafranovshift is missing). Nevertheless, concerning the boundary structures we point out that the upper islands of both sequences intersect the horizontal divertor plates at similar positions. The ergodic structures in the Poincaré plots differ however depending whether they are introduced by the plasma currents or by the vacuum field coils. As will be shown in the next section the difference with respect to the calculated load patterns based on field line diffusion for these different fields are remarkably close. In the experiment, deviations from these results may occur depending on the details of the achievable parameters at the plasma boundary which will need further in- 
vestigations with a SOL-transport code like EMC3/EIRENE which are beyond the scope of this paper. Thus, both effects, $\beta$ - and nettoroidal current, can be combined to simulate the expected heat load distribution of the "SE reference scenario" during the evolution in a long-pulse discharge.

\section{$5 \quad$ Results}

\subsection{Strike-line pattern}

Up to this point, the details of the strikeline patterns have been ignored focussing on the integral power flux onto the components. However, for some locations it is necessary that also the strike-line patterns are similar. This is especially important for the loads near the pumping gap and the loads onto the SE, since the details of their distribution will have a strong influence on the pumping efficiency of the neutrals created on the targets by the outflow of plasma.

Figure 9 compares the strike line pattern of the configuration of the SE-reference scenario with $22 \mathrm{kA}$ (for which an overload at the pumping gap would be expected without the $\mathrm{SE})$ with the pattern generated by the corresponding mimic vacuum configuration. Note that the shape of the pattern on the SE is very similar for the two cases, although for the vacuum case, the hot-spot at the front (upper part in picture) is somewhat more intense. The heat-flux to the pumping gap is at the same level for the two configurations, but for the mimic vacuum field, the horizontal target plate (right part of TDU in picture) is loaded somewhat more.

The strike line pattern for the stationary configuration with $43 \mathrm{kA}$ is shown in Figure 10 along with the corresponding mimic configuration. In this case, the parallel heat flux to the SE is also very similar for the two configurations. The heat load pattern of the full scenario calculation has one more strike line on the horizontal target plate than its corresponding vacuum configuration. This strike line is caused by the additional changes in the island shape due to the $\beta$-effects. With the available vacuum field coil set it was so far not possible to simultaneously provide a perfect match of the changes in the strike line patterns due to $\beta$ and net-toroidal current everywhere. However, since the additional strike line is located far away from the pumping gap, its impact on the pumping efficiency is considered to be very small, so that investigations of the effect on the pumping efficiency should not be affected.

\subsection{Further use of the configu- rational flexibility}

The approach described here opens up more possibilities for generating other testconfigurations. Due to the limits that are set for OP1.2 with respect to the combination of discharge length and heating power it might be useful to be able to additionally vary the heat load on the $\mathrm{SE}$ via adjustments of the magnetic configuration. We give here two examples of magnetic configurations that might be useful for the TDU-SE physics program.

First, the use of the planar coils to vary the horizontal plasma position makes it possible to vary the heat load onto the SE without changing the input heating power. Figure 11a shows the heat loads on the different divertor parts for the mimic-SE-reference configuration being slightly shifted outward with the effect of reducing the load on the SE. This, on the other hand, means that there is a higher risk for overloading the baffle plates on the outward side.

Second, the coil currents in the modular coils can be used to change the magnetic field strength along the axis which is usually larger at the bean-shaped planes (shown in 


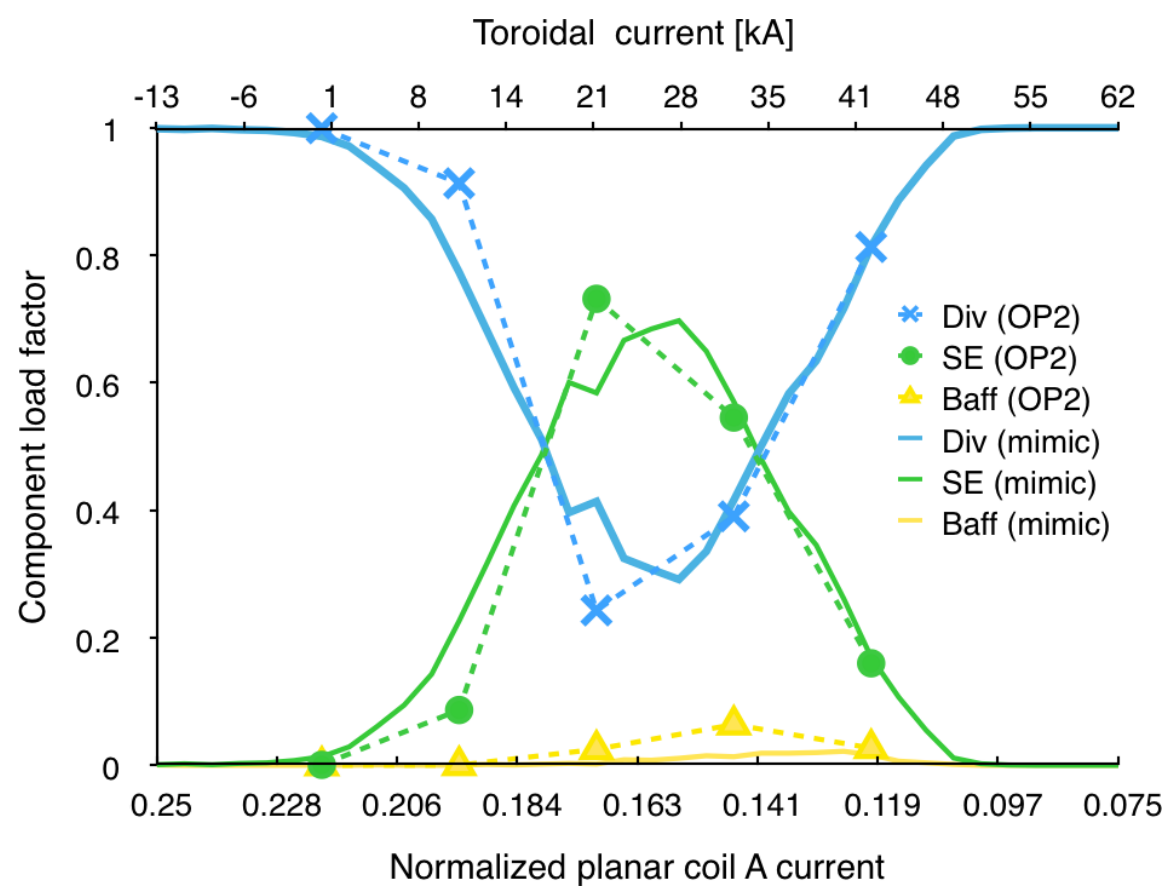

Figure 7: Mimicking the "SE reference scenario" evolution. Lines: planar coil current scan, vacuum configuration. Crosses/Circles/Triangles: MHD-equilibrium calculation of different toroidal currents of the "SE reference scenario". The y-axis shows the fraction of the heat load going to the various components for the different configurations. Mimic configurations: the current of planar coil B is the current in planar coil A minus 0.3. Mimic configurations: Control coil $1=-0.015$, Control coil 2 current $=0.015$. Modular coil currents: $I_{1}=0.96, I_{2}=0.95, I_{3}=0.97, I_{4}=1.07, I_{5}=1.08$. To achieve a main field of $2.5 \mathrm{~T}$ the normalized currents have to been multiplied by $1.47 \mathrm{MA}$.
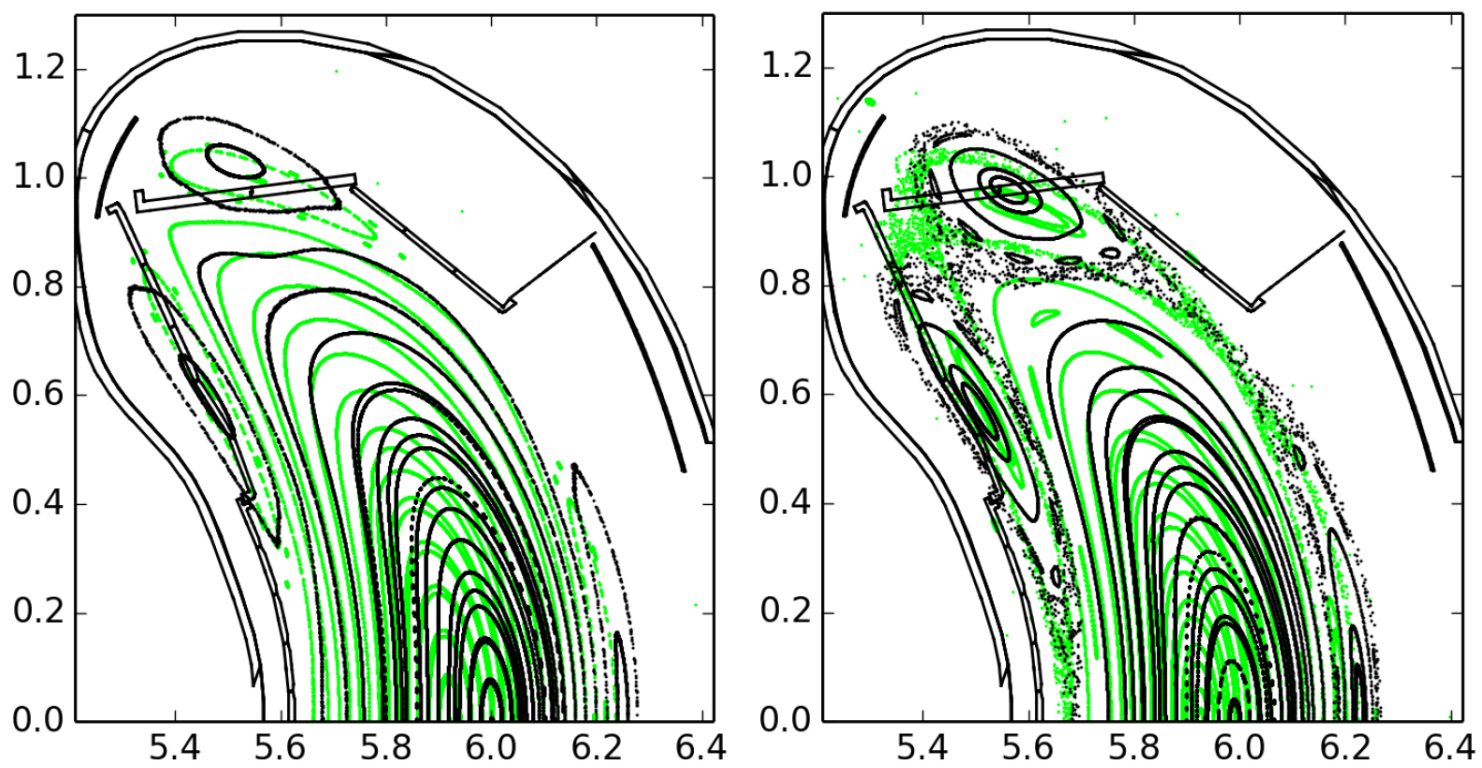

Figure 8: Black: Poincaré plots of vacuum fields of mimic configurations at $\phi=0^{\circ}$. Currents to mimic: (a) $22 \mathrm{kA}$. (b) $43 \mathrm{kA}$. Green: For comparisons SE-reference scenario: (a) $22 \mathrm{kA}$. (b) $43 \mathrm{kA}$. 


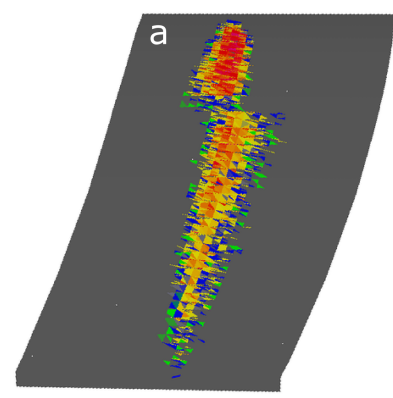

$0 \mathrm{MW}^{2}$

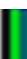

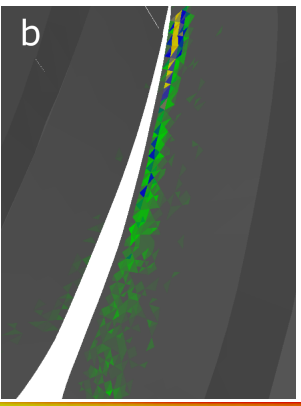

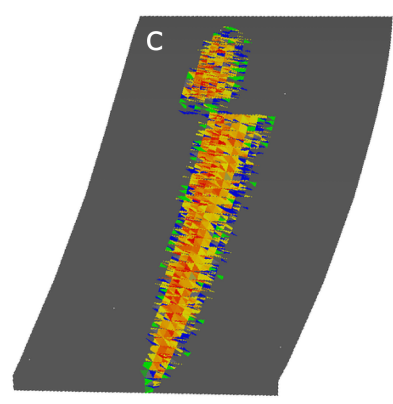

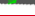

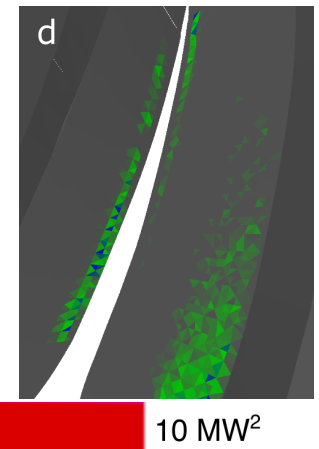

Figure 9: Strike line patterns for the $22 \mathrm{kA}$ configuration, as well as for the corresponding OP1 mimic configuration. (a) SE, mimic configuration/OP1, (b) Divertor, mimic configuration/OP1, (c) SE, "SE reference scenario"/OP2, (d) Divertor, "SE reference scenario"/OP2.
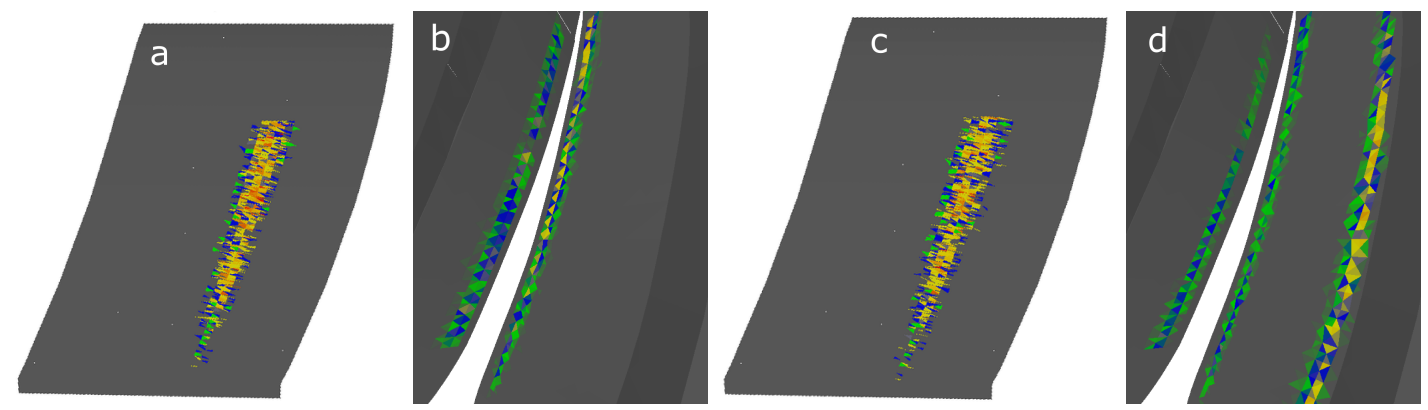

Figure 10: Strike line patterns for the $43 \mathrm{kA}$ steady state configuration, as well as for the corresponding OP1 mimic configuration. (a) SE, mimic configuration/OP1, (b) Divertor, mimic configuration/OP1, (c) SE, "SE reference scenario"/OP2, (d) Divertor, "SE reference scenario"/OP2.
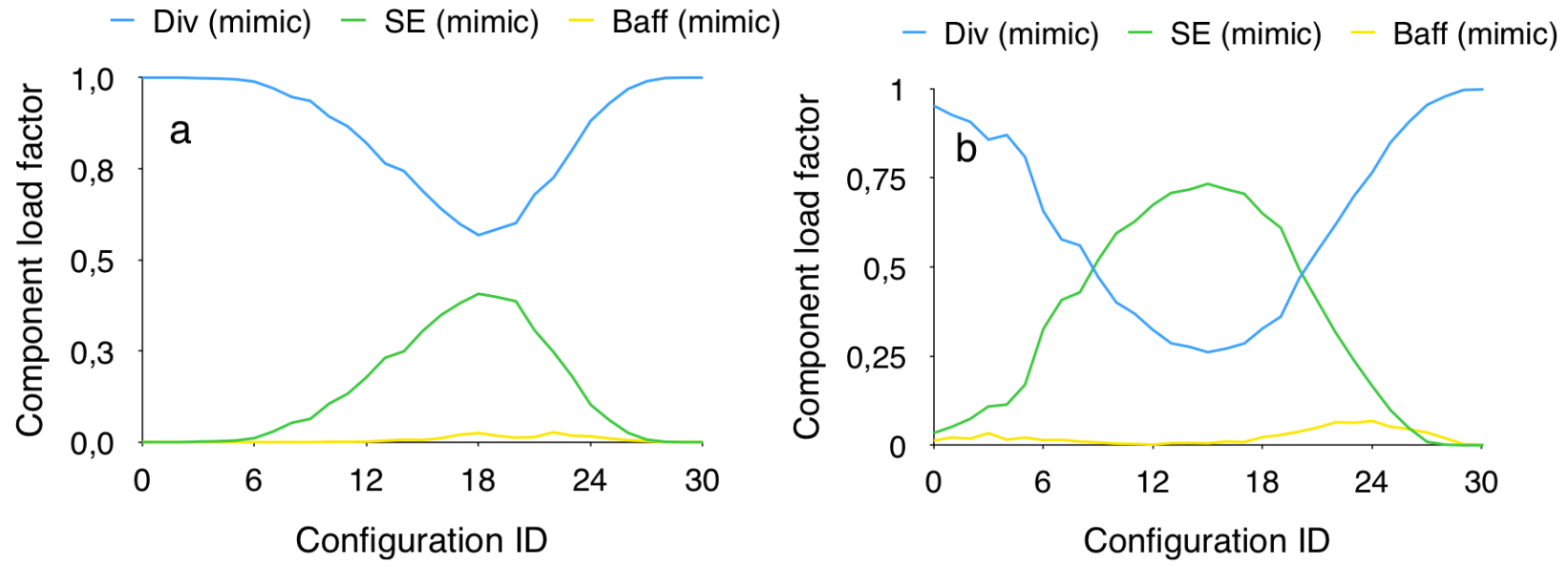

Figure 11: Alternative TDU-SE test scenarios. (a) Reduced parallel heat flux at the SE in a less inward shifted configuration. (b) Increased parallel heat flux at the SE for a configuration with increased mirror ratio. 
the Poincaré plots in the paper) and smaller in the planes in between. Figure 11b shows that by decreasing the field strength between the bean-shaped planes, i.e. increasing the mirror field, it is possible to increase the relative heat load on the SE in the same toroidal current scan as in Figure 11a.

\section{Two versus 10 scraper elements}

As already pointed out, one or two TDU-SE elements will be installed after the first half of the experimental campaign OP1.2, i.e. after OP1.2a. The reduced number - the full set would require 10 SEs - is to limit the effort in manufacturing and in assembly for this first test. Although this introduces an asymmetry in the heat loads, it also offers opportunities for valuable comparisons. On the one hand, it will be possible to compare configurations and discharges without and with SEs installed (OP1.2a vs OP1.2b). On the other hand, the reduced set of SEs in OP1.2b allows a comparison of shielded and unshielded divertor units in the same discharge and configuration. However, for the latter symmetry and periodicity of the magnetic field and of the in-vessel components are crucial conditions, but this will and needs to be investigated in any case.

In case that two TDU-SE elements are installed, the boundary- $\iota$ value of 1 for the configurations we consider here and the argument of stellarator-symmetry suggests to install them close as possible to $180^{\circ}$ toroidally and poloidally separated from each other (one at the top, one at the bottom divertor). For configurations with a boundary-value of $\iota=$ 1 , these two locations are magnetically linked and will shadow each other (and their respective two divertor units). In a full installation there would be 5 (periodicity) such linked divertor and SE combinations. Thus, it is important to know how accurately such a par- tial installation (one or two SE) can be used to assess how the full system of ten scraperelements will behave in later operation. We will focus on two time points during the time evolution of the "SE reference scenario": i.e. when $22 \mathrm{kA}$ of net toroidal current is reached (design point of the SE to avoid the overload of the divertor at the pumping gap) and when $43 \mathrm{kA}$ of net toroidal current is reached - the steady state situation. To investigate the situation to be expected in OP1.2, we base our analysis on the two corresponding mimic vacuum configurations. An extrapolation of the obtained results in this model to what is expected in OP2 with a full set may be nevertheless justified on the basis of the prior results, namely, that the heat load patterns and the expected pumping efficiencies between the mimic configurations and their OP2 counterparts can be expected to be essentially similar. Because for the considered magnetic configurations the boundary structures have the same periodicity as the divertor units, the ones without a SE installed adjacent to them see only a negligible influence from scraper elements installed somewhere else. The heat load onto such units decreases by only $1.5 \%$. The two divertor units with adjacently installed SEs see a similar heat load reduction whether two or ten SEs are installed. The additional heat load reduction when going to the full set of SEs is only $10-12 \%$ of the already reduced heat loads for the $22 \mathrm{kA}$-configuration and almost no effect is seen for the $43 \mathrm{kA}$-configuration. The results are shown in detail in Figure 12. In the case of two SEs, the elements are installed in module 1 at the upper and in module 4 at the lower divertor unit. When no SEs are installed, each divertor unit has a heat load factor of 1 , that is, $1 / 10$ of the total heat load carried by the plasma to all plasma-facing components ends on this divertor module. Analogously, a heat load factor of 0.4 for a particular divertor module means 

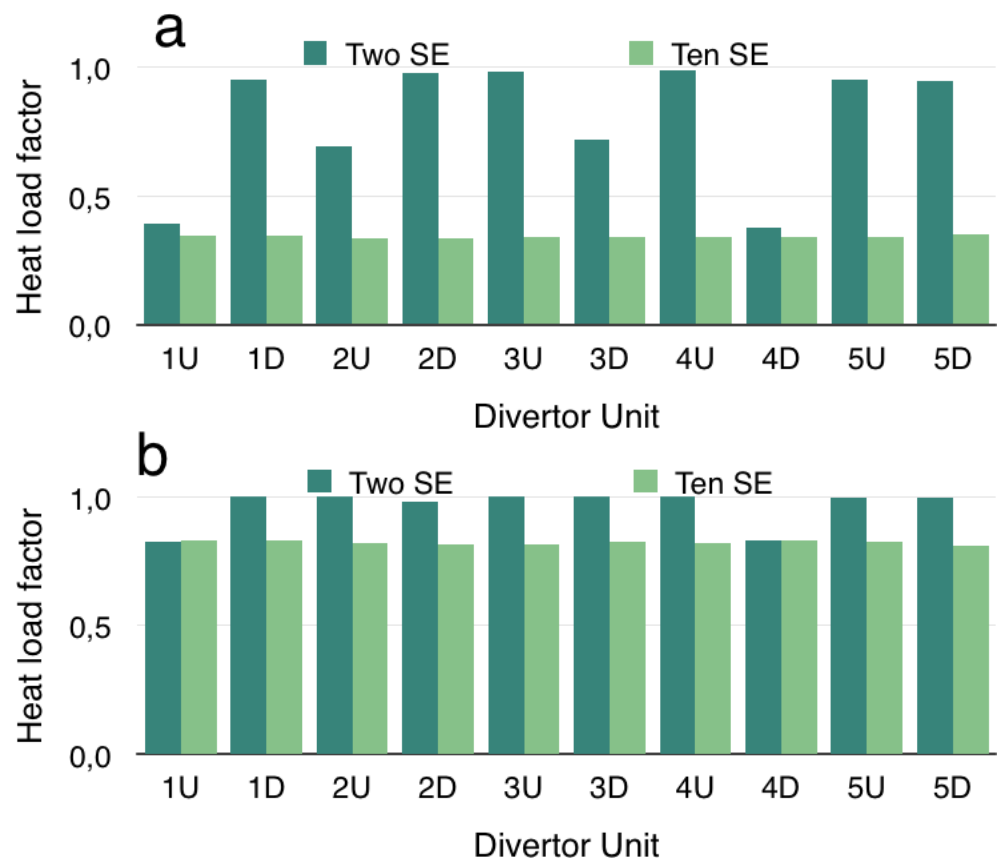

Figure 12: Load onto the different TDU modules comparing reduced (2) and full (10) SE-set. Naming of divertor units: $\mathrm{XY}$ with $\mathrm{X}=$ number of machine module $(1, \ldots, 5)$ and $\mathrm{Y}=$ location within the respective module (U/D=upper/lower divertor unit). (a) OP1.2 configuration for mimicking $22 \mathrm{kA}$ toroidal current. (b) OP1.2 configuration for mimicking $43 \mathrm{kA}$ toroidal current.
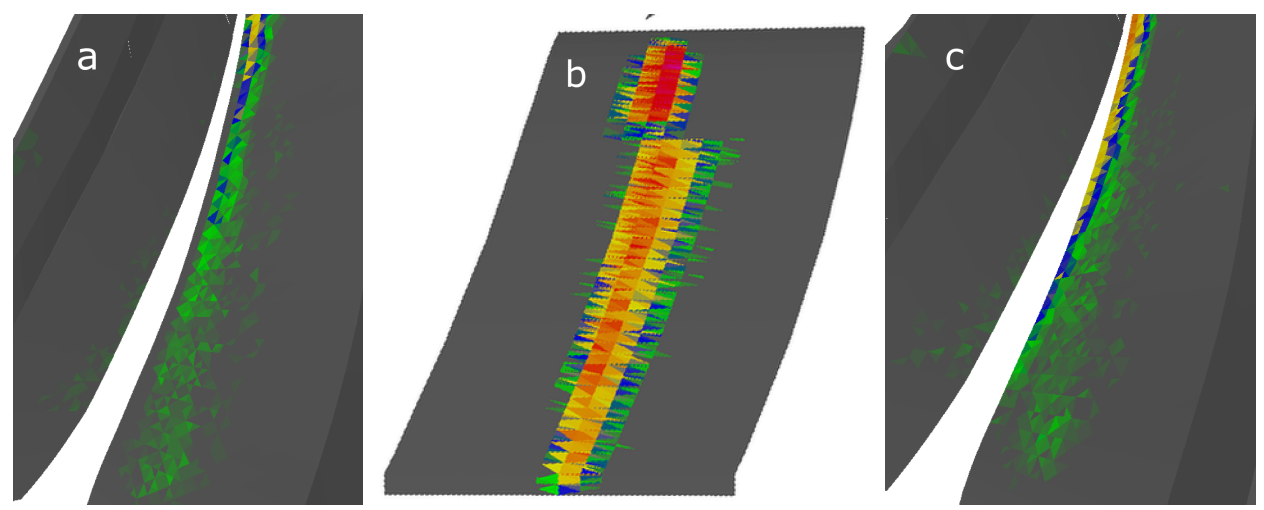

Figure 13: 22kA mimic configuration. Heat load patterns at different divertor parts with only two SE: (a) shielded pumping gap (divertor unit with adjacent SE), (b) SE, (c) unshielded pumping gap (divertor unit without adjacent SE). 
that this module absorbs $40 \%$ of the heat load it would have absorbed in the absence of scraper elements, i.e. $60 \%$ of the heat load is absorbed by one or more scraper elements. Such scraper elements are not necessarily physically located adjacent to the divertor unit that they shield - see for example divertor $2 \mathrm{U}$ in Figure 12a.

The heat loads onto the TDU SEs themselves are, however, substantially different whether two or ten are installed. The heat load onto one of ten SE is about $35 \%$ smaller compared with the heat load at one SE out of two. The reason for this is that the SEs do not only shield their adjacent TDU-module and their magnetically connected counterpart but also SEs in other periods. Also, the effect on the TDU-modules is always distributed onto ten modules (or eight if one considers the unshielded ones), while for the SEs the distribution of the load varies from two to ten. In addition,the strike line pattern on the SE is extended in the direction away from the divertor unit. See Figure 13.

\section{Conclusion}

This paper demonstrates that the available auxiliary coils can be used to mimic complicated plasma effects on the edge topology of W7-X. As presented, our results are somewhat specific to W7-X, although the ansatz to use external coils to simulate effects of plasma currents with respect to the plasma boundary is rather general, e.g. the well-known use of a vertical field to control the horizontal plasma position and thus counteract the Shafranov-shift of the entire plasma column. However, the specifics in our case are on the one hand the coil system of W7-X, i.e. the existence of the planar coils and the sweep coils which have been designed for the purposes we used them for, i.e. iota-control and altering the boundary island properties, and on the other hand the use of the island divertor concept which is connected to the low-shear property of the configuration and susceptibility to rather small net-currents. The results can thus be easily translated to other lowshear devices using the island divertor concept while for other divertor concepts such as the helical divertor in high-shear devices like heliotrons/torsatrons, e.g. LHD, our results cannot be transferred directly.

We have shown that topological changes in the edge region due to $\beta$ and due to net toroidal currents can be mimicked quite similar with near-zero $\beta$, near-zero bootstrap current configurations, using the existing coil set of W7-X. Specifically, effects caused by the MHD-equilibrium currents can be mimicked by adjusting the currents in the sweep coils, and effects caused by the net-toroidal current can be mimicked by adjusting the currents in the planar coils. Thus it seems possible to experimentally investigate aspects of the interaction between plasma and divertor expected in some high-performance plasma scenarios accessible only in the later experimental phase OP2 (2020 and beyond) already in the earlier experimental phase OP1.2 (starting in 2017). Moreover, this allows for an OP1.2 test program for the SEs to assess their potential advantages and disadvantages, despite them being designed to mitigate a possible divertor overload scenario that would only be interesting for quasi-steady-state operation, i.e. for very long discharges, in OP2. Most notable is that for a physics assessment of the effects of the full set of 10 SEs only a subset of two is sufficient to evaluate their effectiveness in configurations with $\iota=1$, thus allowing for significant resource savings. Additionally, the results of such a program can broaden the basis on which a final decision is made whether to manufacture and install a fully cooled SE-set during OP2. It should be noted, however, that the details of the heat load patterns will be somewhat different be- 
tween a situation with ten or with two SEs. Nevertheless, their shielding effect can be assessed accurately enough experimentally and their impact on the pumping-efficiency is expected to be similar enough to be applicable for the other cases. Finally, it should be noted that additional efforts in the scenario development are performed to explore alternatives for the SE-reference scenario avoiding the need of protective measures for the pumping gap.

\section{Acknowledgement}

This work has been carried out within the framework of the EUROfusion Consortium and has received funding from the Euratom research and training programme 2014-2018 under grant agreement No 633053. The views and opinions expressed herein do not necessarily reflect those of the European Commission

\section{References}

[1] Helander, P., Nührenberg, J. "Bootstrap current and neoclassical transport in quasi-isodynamic stellarators" 2009 Plasma Physics and Controlled Fusion, 51(5), 055004. ISO 690

[2] Eich, T. and Sieglin, B. and Scarabosio, A. and Fundamenski, W. and Goldston, R. J. and Herrmann, A. "Inter-ELM Power Decay Length for JET and ASDEX Upgrade: Measurement and Comparison with Heuristic Drift-Based Model" 2011 Phys. Rev. Lett,107,21,215001

[3] Goldston R. J. "Heuristic drift-based model of the power scrape-off width in low-gas-puff H-mode tokamaks" 2012 Nuclear Fusion 52.1 013009. APA

[4] Keilhacker, M., and ASDEX Team. "The ASDEX divertor tokamak." 1985 Nuclear fusion $25.9: 1045$.
[5] Hirsch, M., Baldzuhn, J., Beidler, C., Brakel, R., Burhenn, R., Dinklage, A., Zoletnik, S., et. al. "Major results from the stellarator Wendelstein 7-AS" 2008 Plasma Physics and Controlled Fusion, 50(5), 053001. ISO 690

[6] Grigull, P., McCormick, K., Baldzuhn, J., Burhenn, R., Brakel, R., Ehmler, H., Wenzel, U., et al. First island divertor experiments on the W7-AS stellarator. 2001 Plasma physics and controlled fusion, 43(12A), A175. ISO 690

[7] Spitzer Jr, L. "The stellarator concept" 1958 Physics of Fluids (1958-1988), 1(4), 253-264. ISO 690

[8] Grieger, G., Lotz, W., Merkel, P., Nhrenberg, J., Sapper, J., Strumberger, E., Wagner, F., et. al "Physics optimization of stellarators", 1992 Physics of Fluids B: Plasma Physics (1989-1993), 4(7), 2081-2091. ISO 690

[9] Schmitt, J. C. and Talmadge, J. N. and Anderson, D. T. and Hanson, J. D., "Modeling, measurement, and 3-D equilibrium reconstruction of the bootstrap current in the Helically Symmetric Experiment" 2014 Physics of Plasmas, 092518

[10] Grieger G, Lotz W, Merkel P, Nührenberg J, et al "Physics optimization of Stellarators" 1992 Phys. Fluids B 4, 2081

[11] J.Geiger et al. "Physics in the configuration space of W7-X" 2015 Plasma Phys. Control. Fusion 57014004

[12] Grigull P, McCormick K, Baldzuhn J et al "First island divertor experiments on the W7-AS stellarator" 2001 Plasma Phys. Control. Fusion 43, A175

[13] Bosch H.S, Brakel R, Gasparotto M, et al "Transition From Construction to Operation Phase of the Wendelstein 7-X Stellarator", 2014 IEEE Transactions on Plasma Science, $42,3,432-438$ 
[14] Bozhenkov S.A, Effenberg F, Feng Y, et al "Limiter for the early operation phase of W7-X" 2014 41st EPS Conference on Plasma Physics, P1.080,

[15] Renner H, Sharma D, Kißlinger J, Boscary $\mathrm{J}$, et al "Physical Aspects and Design of the Wendelstein 7-X Divertor", 2004 Fusion Sci. Technol., 46, no.2, 318-326

[16] Boscary J, Peacock A, Friedrich T, et al "Design improvement of the target elements of Wendelstein 7-X divertor", 2012 Fusion Engineering and Design, 87 7-8, 1453-1456

[17] Peacock A, Greuner H, Hurd F, et al "Progress in the design and development of a test divertor (TDU) for the start of W7X operation", 2009 Fusion Engineering and Design, 84, 7-11, 1475-1478

[18] Geiger J, Beidler C.D, Drevlak M, et al "Effects of Net Currents on the Magnetic Configuration of W7-X" 2010 Contrib. Plasma Phys., 50, 8, 770-774

[19] Lumsdaine A, Boscary J, Clark E "Modeling and Analysis of the W7-X High HeatFlux Divertor Scraper Element", 2014 IEEE Transactions on Plasma Science, Part 1

[20] Lore J.D, Andreeva T, Boscary J, et al "Design and Analysis of Divertor Scraper Elements for the W7-X Stellarator" 2014 25th Symposium on Fusion Engineering (SOFE 2013). IEEE.

[21] Helander P., "Theory of plasma confinement in non-axisymmetric magnetic fields", 2014, Rep. Prog. Phys., 77, 087001

[22] Geiger J, Private communication 2013

[23] Hirshman S.P, Whitson J.C "Steepestdescent moment method for threedimensional magnetohydrodynamic equilibria" 1983 Phys. Fluids, 26, 12, 3553-3568

[24] Hirshman S. P, van Rij W. I, Merkel $\mathrm{P}$, "Three-dimensional free boundary calculations using a spectral Green's function method" 1986 Comput. Phys. Commun., 43, 1, 143-155.

[25] Drevlak M, Monticello D, Reimann A, "PIES free boundary stellarator equilibria with improved initial conditions" $2005 \mathrm{Nucl}$. Fusion, 45, 7, 731-740.

[26] Strumberger E "Finite- $\beta$ magnetic field line tracing for Helias configurations" 1997 Nucl. Fusion 37, 1, 19-27

[27] Renner H, Boscary J, Greuer H, et al "Divertor concept for the W7-X stellarator and mode of operation" 2002 Plasma Phys. Control. Fusion 441005

[28] Renner, H., Boscary, J., Erckmann, V., Greuner, H., Grote, H., Sapper, J., Wanner, M., et. al. "The capabilities of steady state operation at the stellarator W7-X with emphasis on divertor design" 2000 Nuclear Fusion, 40(6), 1083. ISO 690

[29] Greuner, H., Bitter, W., Kerl, F., Kisslinger, J., Renner, H. "Structure of divertor for the optimised stellarator W7-X" 1994 Fusion Technology, 323-326. ISO 690

[30] Bozhenkov S.A, Geiger J, Grahl M, et al "Service oriented architecture for scientific analysis at W7-X. An example of a field line tracer" 2013 Fusion Engineering and Design, 88, 11, 2997-3006

[31] Werner A, Endler M, Geiger J, Koenig R. "W7-X magnetic diagnostics: Rogowski coil performance for very long pulses." $2008 \mathrm{Rev}$ Sci Instrum. 79

[32] Rummel T, Risse, K, Ehrke, G. et al, "The Superconducting Magnet System of the Stellarator Wendelstein 7-X" 2012 IEEE Transactions on Plasma Science, 40, No 3, 769-776 